\title{
Human gut microbiome: the second genome of human body
}

\author{
Baoli Zhu ${ }^{1 凶}$, Xin Wang ${ }^{2}$, Lanjuan $\mathrm{Li}^{3}$ \\ ${ }^{1}$ CAS Key Laboratory of Pathogenic Microbiology and Immunology, Institute of Microbiology, Chinese Academy of Sciences, \\ Beijing 100101, China \\ 2 Institute of Plant Protection and Microbiology, Zhejiang Academy of Agricultural Sciences, Hangzhou 310021, China \\ ${ }^{3}$ State Key Laboratory for Diagnosis and Treatment of Infectious Diseases, the First Affiliated Hospital, College of Medicine, \\ Zhejiang University, Hangzhou 310003, China
}

\ Correspondence: zhubaoli@im.ac.cn

Received June 4, 2010 Accepted July 1, 2010

\section{ABSTRACT}

The human body is actually a super-organism that is composed of 10 times more microbial cells than our body cells. Metagenomic study of the human microbiome has demonstrated that there are 3.3 million unique genes in human gut, 150 times more genes than our own genome, and the bacterial diversity analysis showed that about 1000 bacterial species are living in our gut and a majority of them belongs to the divisions of Firmicutes and Bacteriodetes. In addition, most people share a core microbiota that comprises $50-100$ bacterial species when the frequency of abundance at phylotype level is not considered, and a core microbiome harboring more than 6000 functional gene groups is present in the majority of human gut surveyed till now. Gut bacteria are not only critical for regulating gut metabolism, but also important for host immune system as revealed by animal studies.

\section{INTRODUCTION}

For the past decade, human gut microbiota has been extensively studied as more and more scientists believe that human health, apart from our own genome, largely depends on microbes that are living on/in our body (Relman and Falkow, 2001). The term "microbiome", suggested by the Nobel laureate Joshua Lederberg (Lederberg and McCray, 2001; Hooper and Gordon, 2001), refers to the collective genomes of our indigenous microbes or microbiota. Some scientists prefer to call it "metagenome" so as to compare with "metagenomics", which is coined by Handelsman in earlier environmental genomic studies (Handelsman, 2001;
Handelsman, 2004). After the completion of Human Genome Project, in a letter to Science magazine, Julian Davies pointed out that decoding human genome was not enough to understand human biology because there are more than 1000 bacterial species living in and on the human body and they are critically affecting human life. He predicted that these bacteria could harbor 2-4 million uncharacterized genes in addition to the 30,000 genes in human genome, and these two sets of genes could be together determining the human health (Davies, 2001). A few months later, Relman and Folkow called for a second human genome project, to perform a comprehensive metagenomic study of bacterial communities in human mouth, intestinal tract, reproductive tract and skin by random shotgun sequencing to get a thorough understanding of human body (Relman, 2002). However, by 2003 when the human genome was published, the National Institutes of Health $(\mathrm{NIH})$ of United States was not fully convinced, with other large-scale sequencing projects in hand; a large-scale analysis for human microbiome was not initiated until 2007.

Nonetheless, encouraged by the success in a large-scale metagenomic survey by Venter et al. on Sargasso Sea (Venter et al., 2004), other metagenomic studies on human gut microbiota were started worldwide in order to get an insight view on the influence of microbiome over human health. In November 2005, the French National Institute for Agricultural Research (INRA) hosted an international meeting in Paris to discuss a large-scale investigation of human intestinal microbiome. This led to the formation of International Human Microbiome Consortium (IHMC) that aimed to bring international efforts together to establish associations between genes of human intestinal microbiota and human health and disease (Peterson et al., 2009). Supported by 
Seventh Framework Programme in European Union, the main research work was performed under the project named "MetaHIT" (Metagenomics of the Human Intestinal Tract, www.metahit.eu). After the Paris meeting, as part of IHMC, $\mathrm{NIH}$ of the United States officially launched the Human Microbiome Project (HMP, http://nihroadmap.nih.gov/hmp/) in 2007, an even larger effort to study human microbiome in at least four body sites: gastrointestinal tract, mouth, vagina and skin (Turnbaugh et al., 2007; Peterson et al., 2009). The goals of HMP are to sequence 1000 new reference bacterial genomes that are commonly found in human intestinal tract, and to sequence the entire human microbiome for 250 "normal" individuals in 5 years, from 2009 to 2014.

\section{DIVERSITY OF HUMAN INTESTINAL MICROBIOTA}

It is estimated that human intestinal microbiota contains 10 times more bacterial cells than the body cells (Savage, 1977). Initial studies showed that human intestinal microbiome might be composed of 100 times more genes than human genome (Bäckhed et al., 2005). In an attempt to decipher the bacterial diversity of human gut microbiota, a group of scientists from Stanford University performed the first comprehensive diversity analysis using $16 S$ rDNA sequencing approach. In this study, Eckburg et al. (2005) analyzed 13,355 prokaryotic rRNA gene sequences from mucosal tissue and fecal samples of 3 healthy adult people, of which 11,831 were bacterial and 1524 are archaeal. The results showed that the 11,831 bacterial $16 \mathrm{~S}$ rDNA sequences belonged to 395 bacterial phylotypes or bacterial species, and all 1524 archaeal 16S rDNA sequences belong just to one single phylotype, the Methanobrevibacter smithii (Eckburg et al., 2005). In the same study, the authors also indicated that about $80 \%$ of species from human gut microbiota have not been cultivated, and $60 \%$ were new species that have never been described before.

Although there are 70 divisions of Bacteria and 13 divisions of Archaea as described to date, the majority of bacterial species are members of two divisions: Firmicutes and Bacteroidetes. That is, of the 395 species, 301 are Firmicutes, 65 are Bacteroidetes and the other 29 are distributed in eight other divisions. These findings confirmed previous reports on gut diversity (Hold et al., 2002; Hayashi et al., 2002; Wang et al., 2003). The low sensitivity of $16 S$ rDNA analysis would underestimate the bacterial diversity of gut microbiota, thus, the nonparametric methods were applied to explore the phylotype richness and provided at least 500 phylotypes in human intestine (Eckburg et al., 2005). This is confirmed by a deeper microbiome cohort study, which revealed 1000-1150 prevalent species in a pool of 124 human gut microbiota samples, and at least 160 species in each gut microbiota sample (Qin et al., 2010).

At the beginning of Human Microbiome Project, it was thought that there could be a "core microbiome" of shared microorganisms or the so called "core microbiota" in guts of different individuals (Turnbaugh et al., 2007). In a large 16S rDNA survey of gut microbiota for 154 individuals, Turnbaugh et al. (2009) showed that no common bacterial phylotypes (species) were observed at an abundant frequency in human guts. For example, an extensive analysis on nearly 10 thousands full length and 2 million partial $16 \mathrm{~S}$ rDNA sequences demonstrated that there is no single bacterial phylotype or species at an abundance (or abundance frequency) of over $0.5 \%$ in all sampled individuals' gut microbiota. However, it implies that there could be shared phylotypes at lower frequency and/or in smaller population. A deep sequencing of the gut microbiota was performed in a study of 124 Europeans' gut samples, and all bacterial species were compared at $1 \%$ coverage (equivalent to $40 \mathrm{~kb}$ in a typical bacterial genome) of the genomic sequences, which is a different method comparing to the conventional $16 S$ rDNA phylogenetic analysis. Using this approach, 18 species were identified in all individuals and 57 species in $90 \%$ individuals of the same population (Qin et al., 2010). However, further investigation of abundance frequency of these common species in different individuals revealed that there is a high inter-individual variability, ranging from 10 to 2200 folds. This suggests that there could be a "core microbiota" in human gut within a population of defined size without considering the abundance frequency; but if a cut-off value of abundance frequency is applied, the core microbiota will possibly disappear. It is apparent that more studies are required to define the "core microbiota". Nevertheless, accumulating evidences support that the bacterial diversity in human gut mainly depends on diet, geographic location, human health (immune system) itself and other environmental factors. Ley et al. (2006) extensively discussed the determinant factors that influence the diversity of human microbiota.

\section{THE COMPLEMENTARITY OF HUMAN INTESTINAL MICROBIOME}

It is well known that there are 10-100 trillion microorganisms in human intestinal microbiota, and this enormous number presents a huge challenge for scientists to sequence the entire microbiome, especially by conventional Sanger sequencing method. The first attempt to sequence human microbiome was performed by scientists in the Institute for Genomic Research, who used shotgun sequencing approach to sequence DNA samples obtained from fecal specimens of two healthy adults aged 28 (female) and 37 (male). A total of 139,521 high-quality Sanger sequencing reads, comprising nearly 78 million base pairs of unique DNA sequence, were generated for the combined distal gut microbiome of the two subjects, of which 17,668 contigs were assembled and 50,164 open reading frames (ORFs) were predicted (Gill et al., 2006). 
For an extensive comparative functional analysis of this combined human gut microbiome, the authors performed KEGG (Kyoto Encyclopedia Genes and Genomes) pathway analysis and COGs (Clusters of Orthologous Groups) functional analysis. As expected, among the $11 \mathrm{KEGG}$ reconstructed metabolic pathways for distal gut microbiome, genes for polysaccharides metabolism are highly enriched. The COGs functional genes analysis also confirmed these results, indicating that human gut microbiota somehow help the host process ingested carbohydrates, which are probably not digestible by ourselves. The COGs analysis also revealed that a variety of gene groups involved in biosynthesis of essential amino acids and vitamins are enriched, implying the complementarity of gut microbiota to human health. Other enriched genes include those key genes in methanogenic pathway for removing hydrogen gas from human distal gut, and enzymes for detoxication of xenobiotics in human gut (Kurokawa et al., 2007). Our own body would never be able to do these without the assistance of gut microbiota. Removal of hydrogen gas produced during gut bacterial fermentation is very important for efficiently processing dietary polysaccharides, which keeps gut homeostasis in a healthy state.

Almost simultaneously, a Japanese group started an extended comparative metagenomic analysis on human gut microbiome for 13 subjects. Aiming to have a detailed view about the effects of different factors, such as age, diet and host genotype, on the structure and functional capabilities of gut microbial communities, the 13 healthy human subjects were selected from both sexes with ages ranging from 3 months to 37 years old. The total amount of shotgun sequence reads for the 13 gut DNA samples reached 1.06 million, with a read length of $727 \mathrm{Mb}$ and 81,968 assembled contigs, and 662,548 ORFs were predicted based on the MetaGene analysis. The functional analysis of different gut microbiomes identified 3268 COGs, among which 237 COGs are enriched in both adults and children, and 136 COGs are enriched in unweaned infant (Kurokawa et al., 2007). Detailed analysis confirmed pervious findings. For example, genes involved in carbohydrates metabolic pathway and biosynthesis of vitamins and xenobiotics detoxication are highly enriched, which supports the complementarity of gut microbiome. Moreover, the commonly enriched COGs present evidences for a "core microbiome" since the 237 COGs are present in all 9 adults and children (even though this number is not statistically significant). Genes for antimicrobial peptides and multidrug efflux pump are also enriched, which may play a pivotal role in the stable colonization of gut microbes in adult intestine by either producing antimicrobial peptides to kill competitor bacteria or conferring drug resistance to avoid attack.

It is of particular interest to indicate that this study revealed a surge of enriched genes for mobile genetic elements in the intestinal gene pool of 13 Japanese subjects and 2
American subjects from previous work (Gill et al., 2006). These Tn1549-like conjugative transposons (CTns) containing genes for $\mathrm{ABC}$-type multidrug transport systems and Tn916-like CTns harboring integrase/site-specific recombinase, are remarkably expanded in most gut microbiomes, indicating that the horizontal gene transfer (HGT) in human gut microbiota is highly active. It is, therefore, logic to assume that the conjugal elements, which mediate genetic exchanges and transmittance through cell contact, are key players in HGT in human colon. In a separate study, Hehemann et al. (2010) found that porphyranases, a specific group of enzymes in carbohydrates metabolic pathway that process porphyran from marine red algae, are most likely acquired by human gut microbiota through horizontal gene transfer from a marine microbes. These carbohydrate-active enzymes (CAZymes) genes are absent in known terrestrial microbial genomes, but present in several marine bacterial genomes, and are surprisingly found in a human gut bacterium Bacteroides plebeius isolated exclusively from intestinal microbiota of Japanese individuals (Kitahara et al., 2005). Furthermore, an extensive analysis on human microbiome data (Gill et al., 2006; Kurokawa et al., 2007; Turnbaugh et al., 2009) also showed that the porphyranases genes are only present in Japanese gut microbiome. These results are concordant with the fact that Japanese have the tradition of eating raw seaweed food, which could carry marine bacteria and bring the porphyranases genes into human gut. The subsequent horizontal gene transfer possibly occurred to integrate these genes into the genome of Bacteroides plebeius.

\section{CORE MICROBIOME, GENE CATALOG, OBESE AND DISEASE GUTS}

In the Nature paper, Peter Turnbaugh described the "core microbiome" at two different levels: the gut microorganisms and the functional gut genes. For many scientists, the "core microbiota" refers to the core microbiome of commonly shared gut microorganisms, and the "core microbiome" refers to the core microbiome of commonly shared functional gut genes. The confusion comes from the original definition for microbiome. In Scientist journal, Joshua Lederberg defined the microbiome as "... to signify the ecological community of commensal, symbiotic and pathogenic microorganisms that literally share our body space." Later, in the first paper describing Human Microbiome Project (Turnbaugh et al., 2007), Peter Turnbaugh used the following sentence to describe the human microbiota and microbiome: "The microorganisms that live inside and on humans (known as the microbiota) are estimated to outnumber human somatic and germ cells by a factor of ten. Together, the genomes of these microbial symbionts (collectively defined as the microbiome) provide traits that humans did not need to evolve on their own." It is obvious that the microbiome defined 
by Lederberg is more like "microbiota", and Turnbaugh's description falls into the definition of "metagenome". It is probably not so important for now to really define "microbiome", but we should be careful when use different terms to represent the same thing: "microbiome", "microbiota" and "micro flora" for the community of microbes in the human gut, "microbiome" or "metagenome" for the collective genomes of microbes, and the derivatives "core microbiota", "core microbiome" and "core metagenome."

To identify the "core microbiome" at genetic level, several attempts have been made to sequence human microbiome on large scale. The comparative metagenomic analysis on 13 Japanese subjects revealed a core microbiome: among the 3268 enriched COGs, more than 200 commonly enriched COGs are present in all 9 subjects (Kurokawa et al., 2007). In another comparative metagenomics on 18 American subjects, the authors included several monozygotic and dizygotic twin pairs, and the Titanium pirosequencing and Sanger sequencing generated approximately 450,000 sequence reads for each individual, with a total of $2.14 \mathrm{~Gb}$ (Turnbaugh et al., 2009). Functional analysis for several common metabolic pathways showed that the most abundant gene groups are CAZymes, which account for more than $2.6 \%$ of the total sequences in all 18 gut microbiomes. After analyzing more than 20 functional categories, $26 \%-53 \%$ of the functional groups at enzyme level are shared by all 18 microbiomes. This "core microbiome" comprises approximately 2142 total orthologous groups. Combined with the data of bacterial diversity of 18 gut microbiota, the authors also showed that individuals with similar taxonomic profiles share similar metabolic profiles. In the comparative functional analysis of lean and obese twin pair's microbiomes, they identified about 500 obesity-related genes (obesity-enriched and obesity-depleted): $75 \%$ obesity-enriched genes are from Actinobacteria and $25 \%$ from Firmicutes. In contrast, $42 \%$ lean-enriched genes (lean-associated genes) are from Bacteriodetes, while there is no lean-enriched gene from Actinobacteria or obesity-enriched gene from Bacteriodetes. These data indicate that the obesity-enriched genes are exclusively from Actinobacteria and Firmicutes, and the leanenriched genes are from various taxonomic groups, which explained the low diversity in obese gut microbiota. The obesity-associated genes are mainly involved in carbohydrate, lipid and amino acid metabolism.

In a larger-scale of metagenomic analysis, Qin et al. (2010) used Illumina GA sequencer to analyze the gut microbiota of 124 European subjects. A total of $576.7-\mathrm{Gb}$ sequence was generated, from which 3.3 million non-redundant microbial genes were assembled and characterized. This number of genes is 150 folds larger than the human genome itself. There are about 536,000 prevalent unique genes for each gut, showing that most genes are shared by all sampled gut microbiomes. The core microbiome (minimal human gut metagenome) is actually 3 times larger than that described by
Turnbaugh: a total of 6313 functional orthologous groups were found in all 124 subjects. Importantly, in an extensive non-redundant catalog of the minimal human gut metagenome, comparative analysis with published bacterial genomes revealed that two types of functional gene groups are present in each gut bacteria genome and in whole core microbiome: the housekeeping genes and gut specific genes. In this same study, the authors also included 14 healthy individuals and 25 IBD patients (21 Ulcerative Colitis and 4 Crohn's Disease) from Spain. Unfortunately, they were only able to show that the bacterial diversity in these 3 types of microbiota is well separated by Principal Component Analysis; thus, the functional gene groups must be different for IBD patients and healthy individuals. Extensive comparative analysis, deep data mining, and especially, more metagenomic data are needed to elucidate functional gene profiles of the disease guts.

\section{IMMUNE SYSTEM OF MUCOSAL AND SFB BACTERIA}

Whether there is a core microbiota in human intestine is still in debate; however, environmental factors have been shown to play a critical role in the colonization of gut bacteria and the community structure in both human and animals (Yin et al., 2010). Other factors that affect gut microbiota are thought to come from the host: immune system, health status, diet, etc. It is undoubtedly important to understand the correlation of the complex bacterial communities with the nutritional and immunological status of host. In fact, recent studies related to the segmented filamentous bacteria (SFB) in mice revealed a fundamental rule for the interaction of microbiota with host. A main point is that the commensal bacteria in gut play unequal roles in host intestinal immune system, and certain defined commensal species are sufficient to influence the intestinal T cell development (Gaboriau-Routhiau et al., 2009; Ivanov et al., 2009). Initial evidence came from the discovery that locations significantly affect the number of Th17 cells in mouse intestine. The B6 mice from Jackson laboratory have less Th17 cells in small intestine than the ones from Taconic Farms; in addition, the development of Th17 cells could be restored by transferring the fecal contents from Taconic Farms mice to Jackson laboratory mice. Further phylogenetic analysis revealed that the gut bacterial compositions of the two B6 mice were almost identical except that SFB was absent in Jackson laboratory mice, suggesting the significant role of SFB (Ivanov et al., 2008). A parallel study conducted by Gaboriau-Routhiau and colleagues in France made similar observations. Gene expression profiles from the mouse colonized with human gut microbiota or laboratory cultured mouse microbiota were different from conventionally raised mice; the "missing link" was a type of spore forming Clostridium, which was later identified as SFB (GaboriauRouthiau et al., 2009). 
It is clear that SFB is an obligated, spore forming indigenous gut bacteria, which was initially discovered by scanning electron microscope in small intestine of a wide range of hosts, including chickens, pigs, dogs, mice, rats and humans. The most distinguishable characteristics of SFB is the striking adherence to surface of mucosal epitheliums around Peyer's patches through a specified "holdfast structure". As a result, a lot of mitochondria and Golgi bodies were found around the attach area in host cells, suggesting that SFB was involved in activation of host immune system (Yamauchi and Snel, 2000). SFB is unculturable under current microbiological techniques; hence the investigations related to the physiological and immunological roles of SFB were mainly conducted with mono-colonized mice. In comparison to human microbiota colonized mice and mouse microbiota, mono-colonization of SFB resulted in upregulation of ilea genes, stimulation of proinflammatory and regulatory $\mathrm{CD}^{+} \mathrm{T}$ cells differentiation (Gaboriau-Routhiau et al., 2009), activation and recruitment of $C D 8 \alpha \beta^{+}, T C R-\alpha \beta^{+}$ intraepithelial lymphocytes (TCR- $\left.\alpha \beta^{+} I E L\right)$, as well as enhancement of the intestinal IgA responses (Umesaki et al., 1995).

Intestinal immune system has dual purposes: protecting host from pathogenic infections and coexisting with commensal microbiota in gut. The intestinal epithelium cells receive the stimulating signals from luminal agents, and in turn release the proinflammatory products, such as chemokines, cytokines and adhesion molecules to activate the adjacent macrophages, dendritic cells (DC) and mast cells. Luminal bacteria and intestinal epithelium cells crosstalk through a group of well-conserved receptors that recognize pathogenassociated molecular patterns (PAMPs) in luminal bacteria, including toll-like receptors (TLRs) and cytosolic nucleotide binding oligomerization domain (NOD)-like receptors (NOD) (Haller and Jobin, 2004). It becomes clear that multiple cell components from Gram-positive and Gram-negative bacteria can activate TLRs (Michelsen et al., 2001; Martinon and Tschopp, 2005). However, SFB-meditated Th17 cell differentiation seems unrelated to TLRs, NODs or ATP pathway (Ivanov et al., 2009); instead, a significant increase in expression of serum amyloid $A$ (SAA) isoforms, such as Saa1, Saa2 and Saa3, was detected in the terminal ileum of SFB colonized mice. SAA was able to induce the differentiation from naive $\mathrm{CD} 4^{+} \mathrm{T}$ cell into Th17 cells at the presence of intestinal lamina propria (LP) DCs, indicating that SAA mediates SFB colonization and subsequently acts on intestinal DCs to influence Th17 cell differentiation (Ivanov et al., 2009).

Taken together, accumulating evidences suggest that SFB tightly adheres to ilea mucosa and Peyer's Patches (PP) and plays a key role in the coordination of intestinal $\mathrm{T}$ cell profile. However, since SFB still cannot be cultured in vitro, many questions remained to be answered regarding to its physiological and functional roles in human gut.

\section{CULTURING THE UNCULTURABLE HUMAN GUT BACTERIA AND THE POTENTIAL FUNCTIONAL STUDY}

Before the development of molecular ecology techniques, such as phylogenetic classification of colonic microbiota by analyzing the small subunit rRNA gene (16S rRNA), fecal bacteria identification mainly relied on cultivation on selective growth medium, confirmed by cell morphology, gram staining, carbohydrates utilization patterns and short chain fatty acid profiles in PYG medium (Wang and Gibson 1993). According to earlier studies, most human fecal bacteria were culturable on pre-produced anaerobic agar. However, this idea was challenged by Langendijk and collogues in 1995, who found that only $15 \%$ DAPI (4'-6-diamidino-2-phenylindole) counts belonged to culturable bacteria by fluorescence in situ hybridization (FISH) with bifidobacterium probes, indicating the limitation of culture-based approaches on analyzing entire microbial populations (Langendjk et al., 1995). This is further confirmed by the recent $16 \mathrm{~S}$ rDNA sequence analysis of entire human gut microbiota (Eckburg et al., 2005).

Due to the inaccessibility of human intestinal tract, feces were routinely used for studying human gut microbiota. Many scientists consider human large intestine as an open continuous fermentation system-food residues enter from one end in small intestine and fecal materials are excreted from the other end. The peristalsis of large intestine pushed the digested materials slowly passing through it, including cecum, ascending colon, transverse colon, descending colon and sigmoid/rectum (Macfarlane and Macfarlane, 2007). Bacteria colonize on the surface of food particles and mucosal layer with a specific growth rate, and serve as inocula for the newly entered food residues. In general, the bacterial population is similar in ascending, transverse and descending colon, and the bacterial diversity at both genus and species levels was observed regarding to the mucosaassociated bacteria and bacterial residents in lumen (Macfarlane et al., 1992; Ahmed et al., 2007). The fecal samples are now considered as a combination of mucosa shed bacteria and a separate luminal population. Thus, an in vitro gut modeling system can simplify the influence of host genetic background on the metabolism of human microbiota community.

With the rapid development of DNA sequencing techniques, comprehensive studies have been performed on the community structure of individual gut microbiota, but few studies can correlate the microbiota community structure with host phenotype due to the lack of an in vitro culture system for unculturable gut bacteria to investigate their physiological functions and fermentation characteristics in large colon. Based on the data obtained from two sudden-death human victims, of which $\mathrm{pH}$ increased along the longitudinal axis of colon, and short chain fatty acid production decreased distally (Macfarlane et al., 1992), a typical three stage continuous 
culture system was established to simulate the nutritional and environmental conditions of human gut (Macfarlane and Macfarlane, 2007). In general, the parameters to mimic the physiological condition of cecum and ascending colon include carbohydrate-rich medium, low operational $\mathrm{pH}$ and high dilution rate, while protein-rich medium, high $\mathrm{pH}$ and low dilution rate were analogous to the sigmoid/rectum and descending colon (Gibson and Wang, 1994; Macfarlane and Macfarlane, 2007). The in vitro simulation is validated by comparing the bacterial populations and fermentation characteristics, such as volatile fatty acid (VFA) concentration (Macfarlane et al., 1998), and later by FISH (Child et al., 2006). We used 454 pyrosequencing method to compare the major bacterial populations between fecal inoculum and in vitro system, and revealed that $\sim 70 \%$ original microbiota at genus level in inoculum can be maintained in in vitro system (unpublished result), suggesting that the in vitro gut modeling system is a practical approach for investigating human gut metabolism and microbiome.

The in vitro model sustains the growth of considerable portion of uncultivated bacteria; indeed, our recent results demonstrated that $50 \%-70 \%$ sequences detected from the in vitro modeling system belonged to the uncultivated bacteria. However, the composition and portion of uncultivated bacteria in the in vitro model system depend on the medium composition. Hence, "uncultivated bacteria" is a confused term with blurred definitions. In the past 20 years, numerous efforts have been made on developing new non-digestible carbohydrates that possess special property in stimulating the growth of beneficial colonic bacteria at targeted region of large intestine. Thus, in vitro gut modeling systems are widely used in the evaluation of carbohydrates with so called prebiotic effect (Gibson and Wang, 1994; Palframan et al., 2002). Accumulating evidences indicate that the different polymerized carbohydrates are able to significantly affect the structure of microbiota communities, and the cross-feeding among intestinal bacterial species is an essential characteristic in the complicated colonic bacterial community. This implies the potential of in vitro gut modeling system on the enrichment of real cultured colonic bacterial phyla and the subsequently study of its functions.

\section{FUTURE STUDIES OF HUMAN GUT MICROBIOME}

It is evident that gut microbiota plays an essential role in human health. However, the complexity of human gut microbiome and the related research progress are beyond our imagination. Until now, we can only see a tip of the iceberg yet. It is apparent that further comparative metagenomic studies are required to understand the impact of gut microbiome on human health, for example, larger number of samples, deeper sequencing and longer sequence reads. Moreover, linking the gut microbiome to human genotypes (e.g., races), ages and diet, geographic locations, is essential to elucidate the role of human microbiota. Identifying the correlation of gut bacterial genes with phenotypes, such as disease or even behavior, will help improve our health status. It can be expected that, in the near future, gene sets (or biomarkers) will be developed to determine the patients' health status by testing the level of vitamin biosynthesis genes, xenobiotic processing genes, essential amino acid producing genes, etc. Such information will optimize the appolication of prebiotics or probiotics in clinical therapy.

At technological level, it is obvious that high-throughput DNA sequencing approach and related bioinformatic tools are urgently needed. Again, about $60 \%$ gut bacteria are unculturable, which makes it impossible to study individual bacterial genome. Therefore, single cell genomics technology, including microfluidic-based technique for sorting and whole genome DNA amplification by multiple displacement amplification (MDA), is valuable to extract genomic information from unculturable bacteria. Moreover, the chemostat has been used for many years to culture the entire human gut microbiota, that although not perfect, it sustained the majority of human gut microbiota according to our recent results (data not published). Further research is required to optimize the nutrition of chemostat to culture interested gut bacteria in groups.

\section{ACKNOWLEDGEMENTS}

The research grant is supported by the National Basic Research Program (973 Program) "The metagenomic study on HBV infected human intestinal tract” with Grant No. 2007CB513000.

\section{REFERENCES}

Ahmed, S., Macfarlane, G.T., Fite, A., McBain, A.J., Gilbert, P., and Macfarlane, S. (2007). Mucosa-Associated bacterial diversity in relation to human terminal ileum and colonic biopsy samples. Appl Environ Microbiol 73, 7435-7442.

Aoi, Y., Kinoshita, T., Hata, T., Ohta, H., Obokata, H., and Tsuneda, S. (2009). Hollow-fiber membrane chamber as a device for in situ environmental cultivation. Appl Environ Microbiol 75, 3826-3833.

Bäckhed, F., Ley, R.E., Sonnenburg, J.L., Peterson, D.A., and Gordon, J.I. (2005). Host-bacterial mutualism in the human intestine. Science 307, 1915-1920.

Bollmann, A., Lewis, K., and Epstein, S.S. (2007). Incubation of environmental samples in a diffusion chamber increases the diversity of recovered isolates. Appl Environ Microbiol 73, 6386-6390.

Child, M.W., Kennedy, A., Walker, A.W., Bahrami, B., Macfarlane, S., and Macfarlane, G.T. (2006). Studies on the effect of system retention time on bacterial populations colonizing a three-stage continuous culture model of the human large gut using FISH techniques. FEMS Microbiol Ecol 55, 299-310.

Davies, J. (2001). In a map for human life, count the microbes, too. Science 291, 2316.

Eckburg, P.B., Bik, E.M., Bernstein, C.N., Purdom, E., Dethlefsen, L., Sargent, M., Gill, S.R., Nelson, K.E., and Relman, D.A. (2005). 
Diversity of the human intestinal microbial flora. Science 308 , 1635-1638.

Gaboriau-Routhiau, V., Rakotobe, S., Lécuyer, E., Mulder, I., Lan, A., Bridonneau, C., Rochet, V., Pisi, A., De Paepe, M., Brandi, G., Eberl, G., Snel, J., Kelly, D., Cerf-Bensussan, N. (2009). The key role of segmented filamentous bacteria in the coordinated maturation of gut helper T cell responses. Immunity 31, 677-689.

Gibson, G.R., and Wang, X. (1994). Enrichment of bifidobacteria from human gut contents by oligofructose using continuous culture. FEMS Microbiol Lett 118, 121-127.

Gill, S.R., Pop, M., Deboy, R.T., Eckburg, P.B., Turnbaugh, P.J., Samuel, B.S., Gordon, J.I., Relman, D.A., Fraser-Liggett, C.M., and Nelson, K.E. (2006). Metagenomic analysis of the human distal gut microbiome. Science 312, 1355-1359.

Haller, D., Jobin, C. (2004). Interaction between resident luminal bacteria and the host: can a healthy relationship turn sour? J Pediatr Gastroenterol Nutr 38, 123-136.

Handelsman, J. (2004). Metagenomics: application of genomics to uncultured microorganisms. Microbiol Mol Biol Rev 68, 669-685.

Handelsman, J., Rondon, M.R., Brady, S.F., Clardy, J., and Goodman, R.M. (1998). Molecular biological access to the chemistry of unknown soil microbes: a new frontier for natural products. Chem Biol 5, R245-R249.

Hayashi, H., Sakamoto, M., and Benno, Y. (2002). Fecal microbial diversity in a strict vegetarian as determined by molecular analysis and cultivation. Microbiol Immunol 46, 819-831.

Hehemann, J.H., Correc, G., Barbeyron, T., Helbert, W., Czjzek, M., and Michel, G. (2010). Transfer of carbohydrate-active enzymes from marine bacteria to Japanese gut microbiota. Nature 464 , 908-912.

Hold, G.L., Pryde, S.E., Russell, V.J., Furrie, E., and Flint, H.J. (2002). Assessment of microbial diversity in human colonic samples by 16S rDNA sequence analysis. FEMS Microbiol Ecol 39, 33-39.

Hooper, L.V., and Gordon, J.I. (2001). Commensal host-bacterial relationships in the gut. Science 292, 1115-1118.

Ivanov, II., Atarashi, K., Manel, N., Brodie, E.L., Shima, T., Karaoz, U., Wei, D., Goldfarb, K.C., Santee, C.A., Lynch, S.V., Tanoue, T., Imaoka, A., Itoh, K., Takeda, K., Umesaki, Y., Honda, K., Littman, D.R. (2009). Induction of intestinal Th17 cells by segmented filamentous bacteria. Cell 139, 485-498.

Ivanov, II., Frutos Rde, L., Manel, N., Yoshinaga, K., Rifkin, D.B., Sartor, R.B., Finlay, B.B., Littman, D.R. (2008). Specific microbiota direct the differentiation of IL-17-producing T-helper cells in the mucosa of the small intestine. Cell Host Microbe 4, 337-349.

Kitahara, M., Sakamoto, M., Ike, M., Sakata, S., and Benno, Y. (2005). Bacteroides plebeius sp. nov. and Bacteroides coprocola $s p$. nov., isolated from human faeces. Int J Syst Evol Microbiol 55, 2143-2147.

Kurokawa, K., Itoh, T., Kuwahara, T., Oshima, K., Toh, H., Toyoda, A., Takami, H., Morita, H., Sharma, V.K., Srivastava, T.P., et al. (2007). Comparative metagenomics revealed commonly enriched gene sets in human gut microbiomes. DNA Res 14, 169-181.

Langendijk, P.S., Schut, F., Jansen, G.J., Raangs, G.C., Kamphuis, G.R., Wilkinson, M.H.F., and Welling, G.W. (1995). Quantitative fluorescence in situ hybridization of Bifidobacterium spp. with genus-specific $16 \mathrm{~S}$ rRNA-targeted probes and its application in fecal samples. Appl Environ Microbiol 61, 3069-3075.

Lederberg, J., and McCray, A.T. (2001). 'Ome Sweet' Omics-a genealogical treasury of words. The Scientist 15, 8.

Ley, R.E., Peterson, D.A., and Gordon, J.I. (2006). Ecological and evolutionary forces shaping microbial diversity in the human intestine. Cell 124, 837-848.

Macfarlane, G.T., and Macfarlane, S. (2007). Models for intestinal fermentation: association between food components, delivery systems, bioavailability and functional interactions in the gut. Curr Opin Biotechnol 18, 156-162.

Macfarlane, G.T., Gibson, G.R., and Cummings, J.H. (1992). Comparison of fermentation reactions in different regions of the human colon. J Appl Bacterial 72, 57-64.

Macfarlane, G.T., Macfarlane, S., and Gibson, G.R. (1998). Use of a three stage compound continuous culture systems to investigate bacterial growth and metabolism in the human colonic microbiota. Microb Ecol 35, 180-187.

Martinon, F., and Tschopp, J.T. (2005). NLRs join TLRs as innate sensors of pathogens. Trends Immunol 26, 447-454.

Michelsen, K.S., Aicher, A., Mohaupt, M., Hartung, T., Dimmeler, S., Kirschning, C.J., and Schumann, R.R. (2001). The role of toll-like receptors (TLRs) in bacteria-induced maturation of murine dendritic cells (DCS). Peptidoglycan and lipoteichoic acid are inducers of DC maturation and require TLR2. J Biol Chem 276, 25680-25686.

Nichols, D., Cahoon, N., Trakhtenberg, E.M., Pham, L., Mehta, A., Belanger, A., Kanigan, T., Lewis, K., and Epstein, S.S. (2010). Use of ichip for high-throughput in situ cultivation of "uncultivable" microbial species. Appl Environ Microbiol 76, 2445-2450.

Palframan, R.J., Gibson, G.R., and Rastall, R.A. (2002). Effect of pH and dose on the growth of gut bacteria on prebiotic carbohydrates in vitro. Anaerobe 8, 287-292.

Peterson, J., Garges, S., Giovanni, M., Mclnnes, P., Wang, L., Schloss, J.A., Bonazzi, V., McEwen, J.E., Wetterstrand, K.A., Deal, C., et al., and the NIH HMP Working Group. (2009). The NIH Human Microbiome Project. Genome Res 19, 2317-2323.

Qin, J., Li, R., Raes, J., Arumugam, M., Burgdorf, K.S., Manichanh, C., Nielsen, T., Pons, N., Levenez, F., Yamada, T., et al., and the MetaHIT Consortium. (2010). A human gut microbial gene catalogue established by metagenomic sequencing. Nature 464 , 59-65.

Relman, D.A. (2002). New technologies, human-microbe interactions, and the search for previously unrecognized pathogens. J Infect Dis 186, S254-S258.

Relman, D.A., and Falkow, S. (2001). The meaning and impact of the human genome sequence for microbiology. Trends Microbiol 9, 206-208.

Savage, D.C. (1977). Microbial ecology of the gastrointestinal tract. Annu Rev Microbiol 31, 107-133.

Suau, A., Bonnet, R., Sutren, M., Godon, J.J., Gibson, G.R., Collins, M.D., and Doré, J. (1999). Direct analysis of genes encoding $16 S$ rRNA from complex communities reveals many novel molecular species within the human gut. Appl Environ Microbiol 65, 4799-4807.

Tap, J., Mondot, S., Levenez, F., Pelletier, E., Caron, C., Furet, J.P., Ugarte, E., Muñoz-Tamayo, R., Paslier, D.L., Nalin, R., et al. (2009). Towards the human intestinal microbiota phylogenetic core. Environ Microbiol 11, 2574-2584.

Turnbaugh, P.J., Ley, R.E., Hamady, M., Fraser-Liggett, C.M., Knight, R., and Gordon, J.I. (2007). The human microbiome project. 
Nature 449, 804-810.

Turnbaugh, P.J., Hamady, M., Yatsunenko, T., Cantarel, B.L., Duncan, A., Ley, R.E., Sogin, M.L., Jones, W.J., Roe, B.A., Affourtit, J.P., et al. (2009). A core gut microbiome in obese and lean twins. Nature 457, 480-484.

Umesaki, Y., Okada, Y., Matsumoto, S., Imaoka, A., Setoyama, H. (1995). Segmented filamentous bacteria are indigenous intestinal bacteria that activate intraepithelial lymphocytes and induce MHC class II molecules and fucosyl asialo GM1 glycolipids on the small intestinal epithelial cells in the ex-germ-free mouse. Microbiol Immunol 39, 555-562.

Venter, J.C., Remington, K., Heidelberg, J.F., Halpern, A.L., Rusch, D., Eisen, J.A., Wu, D., Paulsen, I., Nelson, K.E., Nelson, W., et al. (2004). Environmental genome shotgun sequencing of the Sargasso Sea. Science 304, 66-74.

Wang, X., Gibson, G.R. (1993). Effects of the in vitro fermentation of oligofructose and inulin by bacteria growing in the human large intestine. J Appl Bacteriol 75, 373-380.

Wang, X., Heazlewood, S.P., Krause, D.O., and Florin, T.H. (2003). Molecular characterization of the microbial species that colonize human ileal and colonic mucosa by using $16 \mathrm{~S}$ rDNA sequence analysis. J Appl Microbiol 95, 508-520.

Winitz, M., Adams, R.F., Seedman, D.A., Davis, P.N., Jayko, L.G., and Hamilton, J.A. (1970). Studies in metabolic nutrition employing chemically defined diets. II. Effects on gut microflora populations. Am J Clin Nutr 23, 546-559.

Yamauchi, K.E., Snel, J. (2000). Transmission electron microscopic demonstration of phagocytosis and intracellular processing of segmented filamentous bacteria by intestinal epithelial cells of the chick ileum. Infect Immun 68, 6496-6504.

Yin, Y., Lei, F., Zhu, L., Li, S., Wu, Z., Zhang, R., Gao, G.F., Zhu, B., Wang, X. (2010). Exposure of different bacterial inocula to newborn chicken affects gut microbiota development and ileum gene expression. ISME J 4, 367-376. 